\title{
Rethinking language education in Taiwan: A case of English majors' use of Chinese classifiers in translation
}

\section{$\mathrm{Yu}$, Bin-Bin $\bowtie$}

Department of Applied Foreign Languages, Lunghwa University of Science and Technology, Taiwan, ROC (bbyu@mail.lhu.edu.tw)

Received: 3 November 2014 Available Online: 1 March 2015
Revised: 19 January 2015 DOI: $10.5861 /$ ijrsll.2015.970

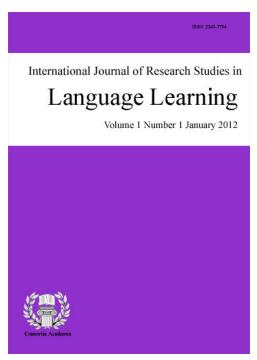

ISSN: 2243-7754 Online ISSN: 2243-7762 OPEN ACCESS

\section{Abstract}

This paper aims to bring attention to language education in Taiwan through a case study of English majors' Chinese proficiency by way of their use of classifiers. Due to the increasing exposure to English learning environments in university, the Chinese proficiency of English learners might be influenced. This study is a first longitudinal investigation into English majors' use of Chinese classifiers in translation. Chinese classifiers are words that occur in the form of a numeral followed by a classifier before a noun the classifier modifies. The lack of awareness of Chinese classifiers causes a variety of problems in English-Chinese translation. English-majored students under investigation were put to tests during their second and fourth years in university with elicitation materials designed by the researcher. Findings show a similar tendency towards the use of Chinese classifiers for the two tests, even misconceptions of some homophonic classifiers.

Keywords: Chinese; classifiers; first language acquisition; second language acquisition; language learning 


\section{Rethinking language education in Taiwan: A case of English majors' use of Chinese classifiers in translation}

\section{Introduction}

With the emergence of English as a global language, the learning of English in Taiwan is booming, especially due to the promotion of the government's educational policy to include English as a subject in Grade 5 in September 2001, and then even in Grade 1 in 2002 (Nunan, 2003). This has even become "a whole-nation movement" (Chern, 2002, p. 104). Many Applied Foreign Languages departments have been established for principally teaching English for communication rather than literature appreciation, particularly in science and technology universities. The instruction of English in these universities essentially involves four skills, i.e. listening, speaking, reading, and writing, and perhaps an additional skill - translating. However, the latter is quite a different matter from the former four in that it involves a good command of both source and target languages. The incorporation of translation courses presents an instant problem of language proficiency of English majors, not only in English but also in Chinese.

Mandarin Chinese is officially taught as a subject from elementary school all the way to the first year of university in Taiwan. When English majors receive limited or no Chinese-language-related education in their later years in university, their Chinese proficiency might be affected due to the increasing exposure to English learning environments (Cook, 2003; Pavlenko, 2000). So far, a wide range of studies regarding language education have paid attention to teaching/learning effectiveness, typically by means of pre- and post-tests during a short period of time. Little research has focused on learning effects for an extended period, especially the effect of something learnt in early childhood - classifiers.

In English, a numeral can quantify a noun by itself, but in Chinese, it cannot do so. When a Chinese noun is preceded by a numeral, a classifier (CL) mandatorily appears directly before the noun (Li \& Thompson, 1981). Thus, while English speakers say 'a fish', Chinese speakers say $y i$ tiao $y u$ 'one $\underline{\mathrm{CL}}$ fish'. The classifier is syntactically obligatory in the noun phrase (NP). The lack of classifiers in source language might cause a problem of neglect in translation, especially word for word (Newmark, 1988). Besides, the selection from a variety of candidate classifiers might also put student translators into trouble. Most Chinese nouns have one or more particular classifiers associated with them. For example, the Chinese noun $y u$ 'fish' can take at least three possible classifiers, namely tiao, wei, and zhi. The choice of one classifier rather than the others depends on language users' awareness. The lack of knowledge of classifiers causes a pragmatic problem. Due to the diverse problems discussed above, an investigation into English majors' use of Chinese classifiers in translation would be necessary.

\subsection{Research objectives}

With the main purpose of the current study to examine English majors' Chinese proficiency by way of their use of classifiers, the objectives of this research are as follows:

$>\quad$ to understand English majors' awareness of the classification of Chinese nouns

$>\quad$ to see if there is any change in using classifiers after an extended period of time

$>\quad$ to find out possible factors that influence students' choice of one classifier rather than the others 


\section{Literature review}

\subsection{L2 influence on L1}

Earlier research on cross-linguistic influence focused mostly on the interference of learners' first language in the additional languages they acquired. This was the case of the classical contrastive analysis and transfer studies (e.g. Lado, 1957; Weinreich, 1953), and also of the later work on interlanguage (e.g. Kellerman, 1983, 1984; Laufer, 1992; Plag, 1992; Zimmermann, 1992; Zobl, 1982). Discussions of research once placed much emphasis on the differences between learners' second language (L2) and their first language (L1). Nevertheless, some attention has been shifted to the perspective of cross-linguistic similarities in L2 learning (Ringbom, 2007).

Recently, a few scholars have noted the influence of L2 on L1 (e.g. Cook, 2003; Jarvis \& Pavlenko, 2008; Pavlenko, 2000). Laufer (2003) examined L2 effects on two areas of L1 lexical competence: the knowledge of collocations and lexical diversity in free written expression. According to her research, the subjects' L1 declined, deteriorated, or suffered attrition to some degree when they acquired an L2 if measured against people who knew only one language. Porte's (2003) investigation was carried out in the context of potential L1 attrition in an L2 environment, and specifically focused on a description of L1 output of long-term-resident EFL (English as a foreign language) teachers whose L1 loss or deviance could be further aggravated by the demands of their everyday working environments. Thus, the increasing dominance of the L2 might be viewed as one of the factors responsible for the gradual disappearance of the L1 (Berman \& Olshtain, 1983; Kaufman \& Aronof, 1989; Weltens, De Bot, \& van Els, 1986). Accordingly, as far as the present study is concerned, students' Chinese proficiency might be affected due to the increasing exposure to English learning environments in university.

\subsection{Definitions}

Classifiers and measure words - Classifiers are commonly referred to as 'measure words', which provide measuring units for noun entities. However, a distinction between these two sets of words should be made. Tai and Wang (1990) defined classifiers as those that categorize "a class of nouns by picking out some salient perceptual properties, either physically or functionally based, which are permanently associated with the entities named by the class of nouns" (p.38) and measure words as those that do "not categorize but denote the quantity of the entity named by a noun" (p.38). This distinction in question has a cognitive basis, and it has been tested on syntactic grounds in Chinese grammar (Tai, 1994). First, while a classifier can be replaced by the general classifier $g e$ without changing the meaning of the expression, a measure word cannot. Thus, the classifier zhang in yi zhang zhuozi 'one table' can be replaced by ge, especially in spoken Mandarin Chinese. In contrast, bang in yi bang rou 'one pound of meat' and qun in yi qun ren 'a crowd of people' cannot be substituted by ge without changing the meaning of the expressions. Second, the modifier marker de can be inserted between a measure word and its head noun but not between a classifier and its head noun (see also Cheng \& Sybesma, 1998). Thus, while the expressions yi bang de rou 'one pound of meat' and yi qun de ren 'a crowd of people' are grammatical, the expression yi zhang de zhuozi is not. Besides, Allan's (1977) early work on classifiers also proposed a similar definition in which "a classifier denotes some salient perceived or imputed characteristics of the entity to which an associated noun refers" (p. 285). In other words, a classifier is used to categorize some characteristics of the entities designated by the nouns and also play the function of categorizing nouns into classes, whereas a measure word is used to measure the quantity of an object or a collection of objects.

Another distinction based on grammar may differ in the usage of terms among scholars (e.g. Cheng \& Sybesma, 1998; Chien, Lust, \& Chiang, 2003; Zhang, 2007). 'Count-noun classifiers', 'count-classifiers', or 'qualifying classifiers' are usually used for "noun entities that can occur naturally in discrete, countable units" (Zhang, 2007, p.44), whereas 'mass-noun classifiers', 'mass-classifiers', 'massifiers', or 'quantifiers' are used "both to quantify those nouns that do not naturally occur in discrete units and as measuring units for concrete nouns" (Zhang, 2007, p.44). Here, the former and the latter terms can find their equivalents respectively in Tai 
and Wang's (1990) definition above. For example, the classifier ben in yi ben shu 'one book' is a count-noun classifier, but the classifiers xiang in yi xiang shu 'one box of books' and ping in yi ping jiu 'one bottle of wine' are mass-noun classifiers. While mass-noun classifiers do not necessarily bear any semantic relationship to the noun with which they are used, count-noun classifiers do (Cheng \& Sybesma, 1998). It is count-noun classifiers that the present study is concerned with.

Categorization of noun classification - As already mentioned, count-classifiers bear some semantic relationship to the noun they modified. Accounts of the semantic relationship can be roughly grouped into classical, prototypical, and arbitrary theories (Ahrens, 1994; Gao \& Malt, 2009; Tai, 1994). The proponents of the classical view held that a count-classifier is associated with a noun only if the noun referent possesses a set of defining properties which serve as "necessary and sufficient criterial conditions" (Tai, 1994, p.4) to form a category. Take the classifier ben as an example. It is used only with nouns referring to objects that are bound into a book-like form such as notebooks, dictionaries, user's manuals, and atlases (Gao \& Malt, 2009).

The prototype theorists proposed that the classification of noun referents can be achieved through association with the prototype or "typicality conditions" (Tai, 1994, p.4). Thus, count-classifiers are associated with a noun that is prototypical of a category, and nouns that have a "family resemblance" (Wittgenstein, 1953) with the prototype noun will take the same classifier. For example, the classifier $k e$ is used for small, three-dimensional, roundish objects like pearls, peas, and soy beans, but other objects such as buttons, bombs, mines, and stars also take $k e$ as their classifier, suggesting that the choice is generalized from the prototype (Gao \& Malt, 2009; Tai, 1994; Zhang, 2007).

Arbitrariness may arise when there is neither set of defining features nor any prototype evoked by the classifier to provide a sense of coherence for the set of entities named by the nouns classified (Gao \& Malt, 2009; Zhang, 2007). For example, the classifier $b u$, commonly used for nouns referring to novels, movies, and telephones, is also applied to nouns referring to vehicles. As a result, the use of one classifier for a wide range of noun entities that share no clear shared prototypical or semantic relationships seems to be learnable only through rote memorization (Gao \& Malt, 2009).

Erbaugh's (1986) research on the historical development of the Mandarin noun classifier system links with child language acquisition and shows how the historical development is paralleled by children's acquisition of the system. She has noted that Chinese classifier use is variable rather than categorical in that the same speaker may alternate among several classifiers, even in a sentence, without any evident meaning or stylistic contrast. This claim seems also to predict the inconsistency of the relationship between nouns and classifiers for the present study. Besides, although specific classifiers are traditionally prescribed for a variety of items, Erbaugh's computational analysis has showed that speakers typically prefer the general to the specified classifiers in verbal discourse.

Some common categories of noun classification have been claimed. The classifier categories are grounded in properties that are believed to be universally salient to human perception (Lyon, 1977; Mithun, 1986). Native speakers of a classifier language are believed to have clear and shared intuitions about what category an object, even new, belong to (Allan, 1977; Hiranburana, 1979). Based on his cross-linguistic study, Allan (1977) has identified seven categories: 1) material, 2) shape, 3) consistency, 4) size, 5) location, 6) arrangement, and 7) quanta. The first four categories are further divided into subcategories. The material category is subcategorized into animacy, inanimacy, abstract and verbal nouns. The shape category is subdivided into one-dimensional, two-dimensional, and three-dimensional (or long, flat, and round). The consistency category is subdivided into flexible, hard or rigid, and non-discrete, and the size category is subdivided into big and small. However, category 5 does not apply to Chinese classifiers, whereas categories 6 and 7 deal with measure words.

Built on Allan's categorization as well as Tversky and Hemenway's (1983, 1984) principle of using attributes derived from parts of objects in human categorization, Tai (1994) has presented five categories: 1) animacy, 2) shape, 3) size, 4) consistency, and 5) attributes referring to parts of objects. However, these umbrella 
Rethinking language education in Taiwan: A case of English majors' use of Chinese classifiers in translation

categories are not mutually exclusive. Some noun entities could belong to more than two categories. For example, the Chinese noun $y u$ 'fish' can be categorized into the animacy category for being an animal and the shape category for being long.

Gao and Malt (2009) have suggested that Chinese classifiers can be simply divided into two broad groups: those in which membership is associated with shape attributes such as length or roundness, and those in which membership is associated with multiple shared features and generally restricted to either animate or inanimate entities. However, they have also admitted that the classification could not be objective. Their study has provided a taxonomy of 126 common classifiers and a large sample of the objects classified by each as a tool for their own and future research. The findings of Gao and Malt's comprehensive investigation into the use of Chinese classifiers serves as a good source for the present study to be based on.

\section{Methods}

\subsection{Participants}

This research was designed as a longitudinal study, wherein the primary purpose was to investigate English majors' use of Chinese classifiers in translation at two time points. Besides, it was also designed as a case study. A class of 44 English-majored students enrolled in the evening session of the Applied Foreign Languages department at a university of science and technology in Taiwan was under investigation. They were chosen for easy access to observation due to regular contact with the researcher as their instructor. All of the participants were English majors, and they had received similar language education in Taiwan before they entered the university in September 2008. In the fourth year of university, their English language proficiency was mostly at the lower-intermediate level according to a pretest. Their average age was around 24 years old, ranging from 21 to 33 years old. 38 students participated in the first investigation in June 2010 while they were sophomores, and 43 students took the second test in November 2011 when they were seniors, with an interval of about one and a half years.

\subsection{Instruments}

The elicitation materials used for this investigation into understanding English majors' awareness of the classification of Chinese nouns consisted of two test papers. They were designed on the following considerations: 1) the inclusion of as many different categories as possible in a two-page test paper for fear that the participants might feel disinclined, and 2) the mixture of different categories to prevent responses from interactive effects. The eliciting papers used for this study covered the common categories of noun classification: animate (e.g. animals and humans), inanimate (e.g. buildings and vehicles), and shape attributes (e.g. long, flat, and round noun entities).

The test papers were composed of a short and a longer form. The short version was used for Time 1, which contained 34 English NPs and their incomplete Chinese equivalents. Therein, all of the Chinese translations could be completed by participants' filling in the blanks only with count-classifiers, shown as below:

$$
\text { two novels_ liang ___ xiaoshuo }
$$

The long form was designed for Time 2, which comprised 71 English NPs and their incomplete Chinese equivalents. The long version for Time 2 included the same 34 English NPs from Time 1 and other new ones mixed with the 34 lest the participants might remember them from Time 1 . The focus of the study was on the count-classifiers elicited for the 34 Chinese nouns repeated in the two test papers.

Additionally, pilot tests were also carried out for the reliability and validity of the elicitation materials. The Cronbach Alpha for the short form for Time 1 was computed to be at 0.651, which is considered reliable (Cohen, Manion, \& Morrison, 2007, p. 506). On the other hand, the Cronbach Alpha for the long form for Time 2 was 
computed to be at 0.743 , which is considered quite reliable (Cohen, Manion, \& Morrison, 2007, p. 506). Besides, test-retest reliability was also computed for the answers to the 34 Chinese nouns, and the correlation coefficient was 0.794 .

\subsection{Data collection and analysis}

Data collection was administered by the researcher at the two time points mentioned above. The elicitation materials were given to the participants in the classroom. They read instructions on the eliciting paper and completed the translations with the most appropriate classifiers they thought. Their answers were checked right on the spot to prevent any missing. After collection, data were coded in the datasheet for analysis. Statistical data analyses such as the number and percentage, and chi-square tests were computed. The focus of the present study was on the common part of the two test papers, namely the count-classifiers for the 34 English NPs and their incomplete Chinese equivalents.

\section{Results and discussion}

Results are discussed in terms of the three research objectives: 1) to understand English majors' awareness of the classification of Chinese nouns; 2) to see if there is any change in using classifiers after an extended period of time; 3) to find out possible factors that influence students' choice of one classifier rather than the others.

\subsection{Students' awareness of the classification of Chinese nouns}

Generally speaking, the results parallel those in other early investigations (e.g. Gao \& Malt, 2009), in particular the most frequently used classifiers for categories such as animals, shapes, buildings, and vehicles at Time 1, as shown in Tables 1 to 4, except for the classifiers jian for fangzi 'house' (see Table 3) and tai for youlanche 'tour bus' (see Table 4). As far as the category of animals is concerned, as shown in Table 1, the most frequently used classifier for ma 'horse' is pi (84\%), the most frequently used classifier for muniu 'cow' is tou $(63 \%)$, and the other nouns are mostly modified by $z h i$. There is a difference between the use of $z h i$ and the other two classifiers in that $z h i$ is a general classifier for animals, whereas pi and tou are associated with particular animals. In addition, the classifier tiao is used to modify nouns referring to longness of shape (see Table 2 below).

The category of shape has conventionally been divided into the three main subcategories: longness, flatness, and roundness. These have been replaced by Allan (1977) with one-dimensional, two-dimensional, and three-dimensional. The present study shows parallel findings that most participants used tiao to classify such nouns referring to objects with one dimensional extension in length as rivers (97\%) and fish (66\%) and also that more than one fourth of the participants preferred the default animal marker $z h i$ for $y u$ 'fish' (26\%), as shown in Table 2. As far as flatness is concerned, zhang and shan are typical classifiers for nouns referring to something flat or saliently two-dimensional objects. The Chinese noun referring to tables was categorized by most of the participants with the classifiers zhang $(76 \%)$ and $g e(21 \%)$, as shown in Table 3. Ge is treated as a general classifier with a default value signaling any existent entities (see Tai, 1994; Gao \& Malt, 2009). The noun referring to doors was most frequently classified by shan (84\%), and it was also modified by dao (13\%). As for roundness, the classifier $k e$ was most frequently used by the participants for such saliently three-dimensional noun entities as oranges (79\%) and apples (89\%), and the two Chinese nouns also took $l i$ as their classifier.

With respect to Chinese nouns referring to buildings, the students' first choice for the nouns fangzi 'house' is dong (76\%), as shown in Table 3. Here, it is worth noting their second choice jian; its usage greatly differs from that in Gao and Malt's (2009) findings, in which nouns referring to stores, restaurants, hotels, supermarkets, companies, and the like are associated with jia, whereas jian is used with nouns referring to any rooms such as bedrooms, kitchen, study office, and workshop. 
Rethinking language education in Taiwan: A case of English majors' use of Chinese classifiers in translation

With regard to vehicles, the classifier liang can be used for Chinese nouns referring to all ground vehicles including cars, trucks, tour buses, and motorcycles, whereas the classifier jia is by far the first choice of the participants for classifying the noun feiji 'airplane'. Besides, it is worth noting that the classifier tai is also frequently used, especially for nouns referring to tour buses, as shown in Table 4. This usage is quite different from that in Gao and Malt's (2009) study, in which tai is used for nouns referring to machines normally with a stand or support such as TV sets, computers, and the like.

\section{Table 1}

Classifiers for nouns referring to animals

\begin{tabular}{lllll}
\hline Category & English-Chinese Translation & Classifiers & Time 1 (N=38) & Time 2 (N=43) \\
\hline \multirow{5}{*}{ Animals } & Two beautiful horses & $p i$ & $84 \%(32)$ & $74 \%(32)$ \\
\cline { 2 - 5 } & liang_meilide ma & $z h i$ & $13 \%(5)$ & $21 \%(9)$ \\
\cline { 2 - 5 } & A cow & tou & $63 \%(24)$ & $54.4 \%(31)$ \\
& $y i \_$muniu & $z h i$ & $26 \%(10)$ & $40.4 \%(23)$ \\
\cline { 2 - 5 } & Two dogs & tiao & $16 \%(6)$ & $26 \%(11)$ \\
\cline { 2 - 5 } & liang_gou & $z h i$ & $87 \%(33)$ & $86 \%(37)$ \\
\cline { 2 - 5 } & A sheep & tou & $11 \%(4)$ & $14 \%(6)$ \\
\hline
\end{tabular}

Note. Only the most frequently used one or two classifiers for each noun entity are shown.

\section{Table 2}

Classifiers for noun entities in different shapes

\begin{tabular}{|c|c|c|c|c|}
\hline Category & English-Chinese Translation & Classifiers & Time $1(\mathrm{~N}=38)$ & Time $2(\mathrm{~N}=43)$ \\
\hline \multirow{3}{*}{ Longness } & A river $y i$ & tiao & $97 \%(37)$ & $98 \%(42)$ \\
\hline & \multirow{2}{*}{ A fish $y i \_y u$} & tiao & $66 \%(25)$ & $53 \%(23)$ \\
\hline & & $z h i$ & $26 \%(10)$ & $35 \%(15)$ \\
\hline \multirow{4}{*}{ Flatness } & \multirow{2}{*}{ A table $y i \_z h o u z i$} & zhang & $76 \%(29)$ & $91 \%(39)$ \\
\hline & & $g e$ & $21 \%(8)$ & $9 \%(4)$ \\
\hline & \multirow{2}{*}{ A door $y i \_m e n$} & shan & $84 \%(32)$ & $88 \%(38)$ \\
\hline & & dao & $13 \%(5)$ & $9 \%(4)$ \\
\hline \multirow{4}{*}{ Roundness } & \multirow{2}{*}{ Four oranges si_liuding } & ke & $79 \%(30)$ & $79 \%(30)$ \\
\hline & & $l i$ & $11 \%(4)$ & $7 \%(3)$ \\
\hline & \multirow{2}{*}{ A Fuji apple yi_fushi pingguo } & $k e$ & $89 \%(34)$ & $86 \%(37)$ \\
\hline & & $l i$ & $8 \%(3)$ & $5 \%(2)$ \\
\hline
\end{tabular}

Note. Only the most frequently used one or two classifiers for each noun entity are shown.

Table 3

Classifiers for nouns referring to buildings

\begin{tabular}{lllll}
\hline Category & English-Chinese Translation & Classifiers & Time 1 (N=38) & Time 2 (N=43) \\
\hline \multirow{2}{*}{ Buildings } & \multirow{2}{*}{ Ten houses shi_fangzi } & dong & $76 \%(29)$ & $60 \%(26)$ \\
\cline { 3 - 5 } & & jian & $21 \%(8)$ & $33 \%(14)$ \\
\hline
\end{tabular}

Note. Only the most frequently used two classifiers for the noun entity are shown.

\section{Table 4}

Classifiers for nouns referring to vehicles

\begin{tabular}{lllll}
\hline Category & English-Chinese Translation & Classifiers & Time 1 (N=38) & Time 2 (N=43) \\
\hline \multirow{5}{*}{ Vehicles } & \multirow{2}{*}{ Six cars liu_qiche } & liang & $79 \%(30)$ & $74 \%(32)$ \\
\cline { 2 - 5 } & \multirow{2}{*}{ Two tour buses liang_youlanche } & tai & $13 \%(5)$ & $9 \%(4)$ \\
\cline { 2 - 5 } & \multirow{2}{*}{ Three airplanes san_feiji } & liang & $45 \%(17)$ & $40 \%(17)$ \\
& & jia & $34 \%(13)$ & $51 \%(22)$ \\
\hline
\end{tabular}

Note. Only the most frequently used two classifiers for each noun entity are shown. 
Yu, B.-B.

4.2 Any change in using classifiers after an extended period of time?

The participants' use of Chinese classifiers remained consistent at Time 2. There was no significant change based on chi-square test results except for a little tendency towards the use of the two classifiers: tai for the noun feiji 'airplane' (due to the test value 9.049 larger than the critical value 5.991 at $\alpha=0.05$ with 2 degrees of freedom) and wei for pinqionde wangzi 'poor prince' (due to the test value 8.509 larger than the critical value 7.815 at $\alpha=0.05$ with 3 degrees of freedom), which have been highlighted in Tables 4 and 5, respectively. The consistency in participants' use of Chinese classifiers might suggest restricted influence of increase in hours for learning English in university. This finding might also be limited to the participants, or lower-intermediate English learners.

\subsection{Possible factors that influence students' choice of classifiers}

A variety of factors could have effects on students' choice of one classifier rather than the others. First of all, their choice might be subject to linguistic conventions such as pi for ma 'horse' and tou for muniu 'cow' (see Table 1). The classifier pi used to refer to the pairing relationship between humans and horses in a time period in ancient China, and it has turned into fossilization and become conventionalized by losing its original semantic motivation (Tai, 1994). The classifier tou is usually used for big animals such as cattle and swine.

Next, students' choice might have its cognitive bases, particularly for the category of shape shown in Table 2. For example, longness of shape has been identified as the cognitive basis of the classifier tiao (Tai \& Wang, 1990). In addition, there was an influence of Taiwanese classifier usages such as $l i$, jian, and particularly tai, shown in Tables 2, 3, and 4 respectively. In Mandarin, tai, as mentioned, is used for nouns referring to machines normally with a stand or support such as TV sets, computers, and the like; however, in the Southern Min dialect or Taiwanese, it can be used for nouns referring to both machines and vehicles. Moreover, it is also worth noting the usages of the other two classifiers, namely $l i$ for round noun entities like oranges and apples (see Table 2) and jian for buildings (see Table 3). In Mandarin, the classifier $l i$ is used to refer to relatively small noun entities such as rice, whereas in Taiwanese, it is used for roundish objects of any size. Besides, in Mandarin, the classifier jian is used with nouns referring to any rooms, whereas in Taiwanese, it can be used with a wider range of noun entities such as rooms, buildings, and companies.

\section{Table 5}

Classifiers for humans

\begin{tabular}{|c|c|c|c|c|}
\hline Category & English-Chinese Translation & Classifiers & Time $1(\mathrm{~N}=38)$ & Time $2(\mathrm{~N}=43)$ \\
\hline \multirow{19}{*}{ Humans } & A person $y i \_r e n$ & $g e$ & $100 \%(38)$ & $100 \%(43)$ \\
\hline & \multirow{2}{*}{ Three children san_xiaohai } & $g e$ & $84 \%(32)$ & $84 \%(36)$ \\
\hline & & wei & $8 \%(3)$ & $12 \%(5)$ \\
\hline & \multirow{2}{*}{ A poor man $y i \_$qiongren } & $g e$ & $47 \%(18)$ & $37 \%(16)$ \\
\hline & & $w e i$ & $39 \%(15)$ & $58 \%(25)$ \\
\hline & \multirow{3}{*}{ Three workers san_gongren } & wei & $42 \%(16)$ & $65 \%(28)$ \\
\hline & & $g e$ & $39 \%(15)$ & $26 \%(11)$ \\
\hline & & ming & $16 \%(6)$ & $7 \%(3)$ \\
\hline & \multirow{3}{*}{ Four students si_xuesheng } & $w e i$ & $79 \%(30)$ & $70 \%(30)$ \\
\hline & & $g e$ & $11 \%(4)$ & $12 \%(5)$ \\
\hline & & $\operatorname{ming}$ & $5 \%(2)$ & $16 \%(7)$ \\
\hline & \multirow{3}{*}{ A poor prince $y i$ pinqionde wangzi } & $w e i$ & $66 \%(25)$ & $88 \%(38)$ \\
\hline & & $g e$ & $26 \%(10)$ & $7 \%(3)$ \\
\hline & & $\operatorname{ming}$ & $3 \%(1)$ & $5 \%(2)$ \\
\hline & \multirow{3}{*}{$\begin{array}{l}\text { A Chinese teacher } \\
y i \quad z h o n g w e n \text { laoshi }\end{array}$} & $w e i$ & $87 \%(33)$ & $88 \%(38)$ \\
\hline & & ming & $11 \%(4)$ & $7 \%(3)$ \\
\hline & & $g e$ & $0 \%(0)$ & $5 \%(2)$ \\
\hline & \multirow{2}{*}{$\begin{array}{l}\text { An aged chairman } \\
y i \quad \text { nianmaide zhuxi }\end{array}$} & $w e i$ & $97 \%(37)$ & $95 \%(41)$ \\
\hline & & $g e$ & $3 \%(1)$ & $2 \%(1)$ \\
\hline
\end{tabular}


Further, the choice of the classifiers for humans, namely ge, wei, and ming (see Table 5), might be guided less by syntactic government but more by pragmatic concerns on the part of language users. Socio-economic status and seniority or age might be an important factor in explaining the use of the classifiers. The neutral classifier $g e$ was not as frequently used as the honorific classifier wei in translation or in this written context. The least used ming is a classifier for people of different professions or in formal contexts.

Moreover, the use of the general classifier $g e$ instead of specific ones might implicate difficulty in getting noun entities classified such as biezhen 'brooch', as in Table 6. In terms of the neo-Gricean pragmatic theory, derived from Grice's (1975) co-operative principle, the use of the informationally weaker expression in a set of contrastive semantic alternates Q-implicates the negation of the interpretation associated with the use of the informationally stronger one in the same set (Huang, 2007). Specific classifiers, which denote their semantic relationships with the noun they modify, are informationally stronger ones, compared with the general classifier, which does not. Thus, the use of the semantically weaker classifier $g e$ implicates the negation of the interpretation associated with the use of specific classifiers. In other words, it is likely that the specific classifiers for the noun do not exist or that the noun was difficult to be classified by the participants into any noun categories (for the notion of implicature, see Grice, 1975; Huang, 2007).

Table 6

The use of general classifier ge

\begin{tabular}{llll}
\hline English-Chinese Translation & Classifiers & Time 1 (N=38) & Time 2 (N=43) \\
\hline \multirow{2}{*}{ A brooch $y i \_$biezhen } & $g e$ & $47 \%(18)$ & $35 \%(15)$ \\
\cline { 2 - 4 } & $z h i$ & $13 \%(5)$ & $23 \%(10)$ \\
\hline
\end{tabular}

Note. Only the most frequently used two classifiers for the noun entity are shown.

Finally, it is worth noting the problem of misconceptions of homophonic classifiers: $z h i$ and $k e$, as in Table 7. Therein, although most participants picked out correct classifiers at Time 2, one third of the students still made errors for the noun referring to trees. On the other hand, although the general animal classifier zhi can also be used to refer to single objects such as yi zhi wazi 'one CL sock' and yi zhi shoutao 'one CL glove', it is unacceptable for the context of 'two pencils'. The other two classifiers can refer to objects with a long stick-like shape. According to Gao and Malt's (2009) study, both have been regarded as classifiers for such noun entities as pencils, pens, cigarettes, arrows, and guns. However, although zhi 'stick-like long object' can be used with a wider range of noun entities, e.g. feathers, dances, and troops, zhi with the meaning of tree branches is more limited in use. The findings of possible factors discussed in this section, especially the misconceptions of the two homophonic classifiers, might be limited to the participants here.

\section{Table 7}

Homophonic classifiers

\begin{tabular}{llll}
\hline English-Chinese Translation & Classifiers & Time 1(N=38) & Time 2 (N=43) \\
\hline \multirow{3}{*}{ Two pencils liang_qianbi } & $z h$ 'stick-like long object' $^{\text {zhi 'tree branch' }}$ & $39 \%(15)$ & $56 \%(24)$ \\
\cline { 2 - 4 } & zhi (for animals or one of a pair) & $39 \%(15)$ & $33 \%(14)$ \\
\hline \multirow{2}{*}{ Two trees liang_shu } & $k e$ (for plants) & $63 \%(8)$ & $9 \%(4)$ \\
\cline { 2 - 4 } & $\begin{array}{l}k e \text { (for something small and roundish in } \\
\text { shape) }\end{array}$ & $37 \%(14)$ & $35 \%(28)$ \\
\hline
\end{tabular}

\section{Conclusions}

This paper has reported on a case study of English-majored students' use of Chinese classifiers in translation. The three research objectives mentioned in the introductory section have been achieved, which include 1) to understand English majors' awareness of the classification of Chinese nouns, 2) to see if there is any change in using classifiers after an extended period of time, and 3) to find out possible factors that influence students' 
choice of one classifier rather than the others. The results can be summarized as follows. In general, English majors' awareness of Chinese classifiers parallels that in other early findings (e.g. Gao \& Malt, 2009). The lower-intermediate English learners' use of classifiers remains consistent, and this might suggest limited influence of increase in hours for learning English in university. Possible factors that influenced students' choice could involve linguistic conventions, cognitive associations, Taiwanese influence, pragmatic concerns, and even misconceptions.

The investigation carried out in this study was limited to English-majored participants' classification of 34 common Chinese nouns. A more comprehensive survey should be conducted in the future study. More other Chinese nouns, especially uncommon ones, should be included in the future examination in order to see how students classify them and then to find out if the results of this study can be generalized to it. Besides, the findings of Taiwanese influence on students' use of Chinese classifiers and their misconceptions of homophonic classifiers, which cause problems to translation, deserve special attention in translating pedagogy as well as future research. Furthermore, this research attended to English majors' Chinese proficiency by way of their use of classifiers. Regarding this construction, there is a great difference in translation between the target language, i.e. Chinese, and the source language, i.e. English, as already mentioned in the introductory section. Further investigations into similarities in construction between the two languages might have different findings from the present one.

The contributions of this study lies in the following important aspects. First of all, English majors' awareness of Chinese classifiers has been realized. Second, this study has noted the importance of research on learning effectiveness for an extended period of time. It is also the first longitudinal investigation into English-majored students' use of Chinese classifiers. Third, a variety of possible factors that influenced English majors' choice of Chinese classifiers have been presented. Last but not least, this study has identified the importance of native language, especially for English majors to learn and do English-Chinese translation. With the trend of learning and teaching English fervently in Taiwan, maybe it is time for English language learners and educators to rethink the importance of Chinese language education.

\section{References}

Ahrens, K. (1994). Classifier production in normals and aphasics. Journal of Chinese Linguistics, 22(2), 202-247.

Allan, K. (1977). Classifiers. Language, 53(2), 285-311. http://dx.doi.org/10.1353/lan.1977.0043

Berman, R. A., \& Olshtain, E. (1983). Features of first language transfer in second language attrition. Applied Linguistics, 4(3), 222-234. http://dx.doi.org/10.1093/applin/4.3.222

Cheng, L. L.-S., \& Sybesma, R. (1998). Yi-wan tang, yi-ge tang: Classifiers and mass-classifiers. The Tsing Hua Journal of Chinese Studies, 28(3), 385-412.

Chern, C.-L. (2002). English language teaching in Taiwan today. Asia-Pacific Journal of Education, 22(2), 97-105. http://dx.doi.org/10.1080/0218879020220209

Chien, Y.-C., Lust, B., \& Chiang, C.-P. (2003). Chinese children's comprehension of count-classifiers and mass-classifiers. Journal of East Asian Linguistics, 12(2), 91-120. http://dx.doi.org/10.1023/A:1022401006521

Cohen, L., Manion, L., \& Morrison, K. (2007). Research methods in education. New York: Routledge.

Cook, V. (Ed.). (2003). Effects of the second language on the first. Clevedon: Multilingual Matters.

Erbaugh, M. S. (1986). Taking stock: The development of Chinese noun classifiers historically and in young children. In C. Craig (Ed.), Noun classes and categorization (pp. 399-436). Amsterdam: John Benjamins. http://dx.doi.org/10.1075/tsl.7.25erb

Gao, M. Y., \& Malt, B. C. (2009). Mental representation and cognitive consequences of Chinese individual classifiers. Language and Cognitive Processes, 24(7/8), 1124-1179. http://dx.doi.org/10.1080/01690960802018323

Grice, H. P. (1975). Logic and conversation. In P. Cole \& J. Morgan (Eds.), Syntax and semantics: Speech acts 
(Vol. 3, pp. 41-58). London: Academic Press.

Hiranburana, S. (1979). A classification of Thai classifiers. Southeast Asian Linguistic Studies, 4, $39-54$.

Huang, Y. (2007). Pragmatics. Oxford: Oxford University Press.

Jarvis, S., \& Pavlenko, A. (2008). Crosslinguistic influence in language and cognition. New York: Routledge.

Kaufman, D., \& Aronof, M. (1989). Morphological interaction between L1 and L2 in language attrition. In S.

Gass, C. Madden, D. Preston, \& L. Selinker (Eds.), Variation in second language acquisition (pp.

202-215). Clevedon: Multilingual Matters.

Kellerman, E. (1983). Now you see it, now you don't. In S. Gass \& L. Selinker (Eds.), Language transfer in language learning (pp. 112-134). Rowley, MA: Newbury House.

Kellerman, E. (1984). The empirical evidence for the influence of the L1 in interlanguage. In A. Davies, C. Criper, \& A. Howatt (Eds.), Interlanguage (pp. 98-122). Edinburgh: Edinburgh University Press.

Lado, R. (1957). Linguistics across cultures: Applied linguistics for language teachers. Ann Arbor: University of Michigan Press.

Laufer, B. (1992). Native language effect on confusion of similar lexical forms. In C. Mair \& M. Markus (Eds.), New departures in contrastive linguistics (pp. 199-210). Innsbruck: University of Innsbruck.

Laufer, B. (2003). The influence of L2 on L1 collocational knowledge and on L1 lexical diversity in free written expression. In V. Cook (Ed.), Effects of the second language on the first (pp. 19-31). Clevedon: Multilingual Matters.

Li, C. N., \& Thompson, S. A. (1981). Mandarin Chinese: A functional reference grammar. London: University of California Press.

Lyon, J. (1977). Semantics. Cambridge: Cambridge University Press.

Mithun, M. (1986). The convergence of noun classification systems. In C. Craig (Ed.), Noun classes and categorization (pp. 379-397). Philadelphia: John Benjamins. http://dx.doi.org/10.1075/tsl.7.24mit

Newmark, P. (1988). A textbook of translation. New York: Prentice Hall.

Nunan, D. (2003). The impact of English as a global language on educational policies and practices in the Asia-Pacific region. TESOL Quarterly, 37(4), 589-613. http://dx.doi.org/10.2307/3588214

Pavlenko, A. (2000). L2 influence on L1 in late bilingualism. Issues in Applied Linguistics, 11(2), 175-205.

Plag, I. (1992). 'There was a man picking pears'. Participial ing-clauses in native language, interlanguage, and target language. In C. Mair \& M. Markus (Eds.), New departures in contrastive linguistics (pp. 223-236). Innsbruck: University of Innsbruck.

Porte, G. (2003). English from a distance: Code-mixing and blending in the L1 output of long-term resident overseas EFL teachers. In V. Cook (Ed.), Effects of the second language on the first (pp. 103-119). Clevedon: Multilingual Matters.

Ringbom, H. (2007). Cross-linguistic similarity in foreign language learning. Clevedon: Multilingual Matters.

Tai, J. H. Y. (1994). Chinese classifier systems and human categorization. In M. Y. Chen \& O. J. L. Tzeng (Eds.), In honor of William S.-Y. Wang: Interdisciplinary studies on language and language change (pp. 479-494). Taipei: Pyramid Press.

Tai, J. H. Y., \& Wang, L. (1990). A semantic study of the classifier tiao. Journal of the Chinese Language Teachers Association, 25(1), 35-56.

Tversky, B., \& Hemenway, K. (1983). Categories of environmental scenes. Cognitive Psychology, 15(1), 121-149. http://dx.doi.org/10.1016/0010-0285(83)90006-3

Tversky, B., \& Hemenway, K. (1984). Objects, parts, and categories. Journal of Experimental Psychology: General, 113(2), 169-193. http://dx.doi.org/10.1037/0096-3445.113.2.169

Weinreich, U. (1953). Languages in contact. The Hague: Mouton.

Weltens, B., De Bot, K., \& van Els, T. (1986). Language attrition in progress. Dordrecht: Foris.

Wittgenstein, L. (1953). Philosophical investigation. New York: Macmillan.

Zhang, H. (2007). Numeral classifiers in Mandarin Chinese. Journal of East Asian Linguistics, 16(1), 43-59. http://dx.doi.org/10.1007/s10831-006-9006-9

Zimmermann, R. (1992). Lexical knowledge: Evidence from L1 and L2 negatives and L1-L2 translations. In C. Mair \& M. Markus (Eds.), New departures in contrastive linguistics (pp. 301-311). Innsbruck: 
Yu, B.-B.

University of Innsbruck.

Zobl, H. (1982). A direction for contrastive analysis: The comparative study of developmental sequences. TESOL Quarterly, 16(2), 169-183. http://dx.doi.org/10.2307/3586790 